\title{
A Probabilistic Perspective of Human-Machine Interaction
}

\author{
Mustafa Canan \\ Naval Postgraduate School \\ Information Sciences Department \\ anthony.canan@nps.edu
}

\author{
Mustafa Demir \\ Arizona State University \\ Ira A. Fulton School of Eng. \\ mdemir@asu.edu
}

\author{
Samuel Kovacic \\ Old Dominion University \\ Eng. Management and Systems Eng. \\ skovacic@odu.edu
}

\begin{abstract}
Human-machine interaction (HMI) has become an essential part of the daily routine in organizations. Although the machines are designed with state-of-theart Artificial Intelligence applications, they are limited in their ability to mimic human behavior. The humanhuman interaction occurs between two or more humans; when a machine replaces a human, the interaction dynamics are not the same. The results indicate that a machine that interacts with a human can increase the mental uncertainty that a human experiences. Developments in decision sciences indicate that using quantum probability theory (QPT) improves the understanding of human decisionmaking than merely using classical probability theory (CPT). In this paper, we examine the HMI from a QPT perspective. Applying QPT to studying HMI for decision-making shows improvement in understanding the decision process when interacting with machines because it provides insights into the mental uncertainty of a human that is not apparent in CPT.
\end{abstract}

\section{Introduction}

The advances in artificial intelligence (AI) and machine learning (ML) have created and will continue to create fundamental shifts in human decisionmaking. Although there is considerable evidence supporting the advantage of AI/ML-based systems for decision support [1], in certain situations interacting with a machine can inadvertently affect the human decision process.

According to the national intelligence institute report, there are two types of AI, Artificial General Intelligence (General AI) and Artificial Narrow Intelligence (Narrow AI) [2]. General AI is known as a system that can handle memory, learning, abstraction, and creativity. Since General AI is not feasible soon, the limitations of narrow AI systems on human behavior must be comprehensively studied due to the limitations of CPT. Narrow AI is a system that is specialized for single purposes and cannot be generalized, and this limits studies on multi-purpose, multi-agent situations. Narrow AI is the standard approach and is built as an optimizing machine [3]. All of the most remarkable advances in AI applications use narrow AI [2]. These systems may be large and complicated and are considered closed systems. In situations where HMI is prevalent, it is considered complex and an open system. As a result, an AI-based system that interacts with a human cannot adapt to the mental states or choice behavior of a human as humans adapt to other humans [12]. Since probability theories play a significant role in designing and developing narrow AI systems [4], their limitations can be better captured by understanding the limitations of the utilized probability theories. These narrow AI/ML applications are based on the Kolmogorovian probability theory, also known as classical probability theory (CPT).

In this paper, we investigate the human-machine interaction (HMI) by using a more comprehensive approach to model human behavior, called quantum probability theory (QPT) [5]. By using QPT [5]-[7], we first model human-human interaction while two humans exchanging information; then apply this model to a scenario in which a human reads online medical information and compare the results in the case of the same human visiting a doctor.

\subsection{Human-machine interaction (HMI)}

HMI refers to a rationalization of relevant attributes and categories that emerge from the use of computerized machines. Along with the human factors line, this rationalization is drawn by physical (e.g., human-body related factors), cognitive (e.g., workload), and emotional (e.g., motivation) factors [8]. Cognition in the HMI is centralized within software due to its role in automation how it mediates most of the tasks. This allows us to interact with machines through software to execute a task. Within that interaction, task factors are crucial because the task is organized, supported by the machine, and executed by the human user [9]. This execution is called activity which is the process of the human becomes aware of the environment by acting on it and by transforming it. The interaction with the environment in which the user and machine are also important because it influences the task user and execution; hence, the process of activity (i.e., execution) is linked to environmental complexity [8]. The current HMI mostly considers explicit communication. In this study, we will examine the interaction dynamics between a human and the online medical content by considering relative and implicit information gain by capitalizing on decision-making theories. 


\subsection{Decision-making}

Decision-making refers to identifying and choosing options by comparing with alternatives based on the values and preferences of the decisionmaker in an interactive task environment [10]. According to Baker et al. (2001), decision-making should start with the identification of the decisionmaker(s) in the situation, reducing the possible disagreement and uncertainty about problem definition, requirements, and goals [11]. For instance, a human with medical complaints can visit a doctor and have the doctor diagnose the problem. By interacting with a doctor, the patient communicates explicitly with the doctor; therefore, all symptoms will be discussed and categorized. This interaction allows the human to develop coherent rationales and opinions by directly communicating with the doctor; hence, this decision process involving agent interdependency.

On the other hand, reading online medical information and learning more about the symptoms involves more implicit communication because in this situation, neither the patient nor the webpage can make any explicit categorization of the symptoms (i.e., no explicit communication, no test results) and each missing categorization of the symptoms continue to influence the final decision. Although a more sophisticated AI-based machine could better assist a human and adapt to the questions that are asked by the human and since this can only be a narrow AI, an interdependent decision is not plausible for a machine; therefore, the interdependency that is observed in human-human interaction cannot emerge in HMI, and the ensuing decisions become individual. Traditionally, HMIs are typically modeled with the rules of CPT, involving two utility-maximizing agents [12]. However, in the case of having heterogonous agents, the differences that stem from relative information gain can complicate decision outcomes because, for a machine, the utility for two is not attainable [3]. In this paper, we use quantum decision theory [6], [13], [14] to understand the implications of HMI in the decision-making process. The findings of this paper are based on an interaction that occurs between a human and computer software, which represents the machine.

We address the following two research questions: (1) What is the interdependency relation between information exchanging agents due to the relative information gain? (2) How can the human decision process be expressed probabilistically by using QPT when there is at least one non-human information source?

We hypothesize that due to the situational relations between information exchanging human agents if a machine/computer replaces one of the humans, the uncertainty during the decision process that humans experience (because of the mental indecisiveness) increases.

The structure of the paper is as follows. First, we discuss the two types of probability theories, i.e., CPT and QPT. Then we elaborate on the double-slit experiment in physics and how it can be used to model human decision-making. Next, we explain quantum decision theory and interdependency in decisionmaking between agents that exchange information. Finally, we discuss the application of the quantum decision theory and how that can elucidate arising anxiety while searching online information by using a case discussed in the book, The Cyber Effect [15].

\section{Probability theories}

\subsection{Classical probability theory}

If $\mathrm{P}$ and $\mathrm{N}$ are two independent events, the probability they both occur is the product of their single-event probabilities, $\quad p(P$ and $N)=p(P)$. $p(N)$. When events become dependent events, the probability of event $\mathrm{P}$ happening affects the probability of event N. The "and" rule for dependent events is expressed as:

$$
p(P \text { and } N)=p(P \cap N)=p(P) \cdot p(N \mid P) .
$$

The standard logic [4] with set theory principles indicates that $\mathrm{p}(\mathrm{P}$ and $\mathrm{N})=\mathrm{p}(\mathrm{N}$ and $\mathrm{P})$. The set theory principles also require that the probability of the conjunction (intersection) of two events, $p(P \cap N)$, must be less than or equal to the probability of single events: $p(P \cap N) \leq p(P), p(N)$. Also, the set theory principles require that the probability of the union of two events must be greater than or equal to the probability of single events: $p(P \cup N) \geq p(P), p(N)$. The decision models that capitalize on CPT rules suppose that there is always a definite mind state for decision-makers; the evolution of system state is assumed to be happening from one state to another (Figure 1).

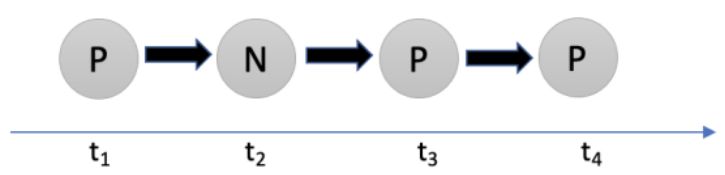

Figure 1. Mental system representation with CPT modeling. The primary assumption is that the system is in a definite state, and it jumps from one state to another. 


\subsection{A CPT violation example: The sure-thing principle}

The sure-thing principle is one of the principles of rational decision-making [16], and it states that "if the alternative $\mathrm{A}$ is preferred to the alternative $\mathrm{B}$, when an event $E_{1}$ occurs, and it is also preferred to $B$, when an event $E_{2}$ occurs; then $A$ should be preferred to $B$, when it is not known which of the events, either $E_{1}$ or $E_{2}$ has occurred. This premise is derived from the rules of CPT, and violation of the sure-thing principle is known as the disjunction effect that violates the union probability $p(A \cup B) \geq p(A), p(B)$ rule of $C P T$. According to rational decision-making principles, the sure-thing principle must always be satisfied in empirical tests. However, systemic violations of this principle have been observed in experiments [5]-[7]; CPT rules fail to explain these systemic violations, whereas QPT coherently explains the sure-thing principle violations [5]-[7].

\subsection{Quantum probability theory}

The paradoxes similar to the sure-things principle are observed when a joint mental representation of events may not be formed [5]-[7], [17]. In this type of situation, the axioms of CPT, (e.g., commutativity transitivity axioms), limit the cognitive models. QPT has been introduced to decision sciences and successfully explained the majority of the paradoxical findings that CPT fails to explain [5]-[7], [17]. QPT removes some strict axioms of the Boolean logic that form the foundations of CPT. It replaces the set logic with the logic of the subspace. In QPT, events are not always commutative, which means $\mathrm{p}(\mathrm{B} \cap \mathrm{A}) \neq \mathrm{p}(\mathrm{A} \cap \mathrm{B})$ is supported [5].

When events are incompatible, a joint mental representation of the events may not be formed. Events can become incompatible when 1) information sources have different views; 2) the perceiver lacks the knowledge or experience to evaluate an event. Under these conditions, the event is evaluated by evoking an incongruent perspective [5]. Since QPT uses a projective probability; hence, incompatible events can be studied in a probabilistic framework.

In QPT, events are represented as subspaces in Hilbert space (Figure 2). For example, a system, S, can be represented by two bases, $\mathrm{P}$ and $\mathrm{N}$, which are the subspaces of a two-dimensional Hilbert space. The cognitive system $\mathrm{S}$ is represented as the superposition of the subspaces $\mathrm{P}$ and $\mathrm{N},|\mathrm{S}\rangle=\mathrm{a}|\mathrm{P}\rangle+\mathrm{b}|\mathrm{N}\rangle$; in this equation are complex numbers; they form the foundation of the probability calculation in QPT. The probability of a decision, $\mathrm{P}$, is calculated by projecting the cognitive state vector $|S\rangle$ on the subspace $|\mathrm{P}\rangle$. A projection operator can be written as $\mathrm{P}_{\mathrm{P}}=|\mathrm{P}\rangle\langle\mathrm{P}|$. If $\mathrm{P}_{\mathrm{A}}$ operates on a superposition vector $P_{P}|S\rangle=$ $|\mathrm{P}\rangle\langle\mathrm{P}|(\mathrm{a}|\mathrm{P}\rangle+\mathrm{b}|\mathrm{N}\rangle)=\langle\mathrm{P}|\mathrm{a}| \mathrm{P}\rangle| \mathrm{P}\rangle$, the inner product is $\langle\mathrm{P} \mid \mathrm{N}\rangle=0$ because of the orthogonality; the probability of event $\mathrm{P}$ is calculated as $\mathrm{p}(\mathrm{P})=$ $|\langle\mathrm{P}|\mathrm{a}| \mathrm{P}\rangle|^{2}=\mathrm{a}^{2}$.

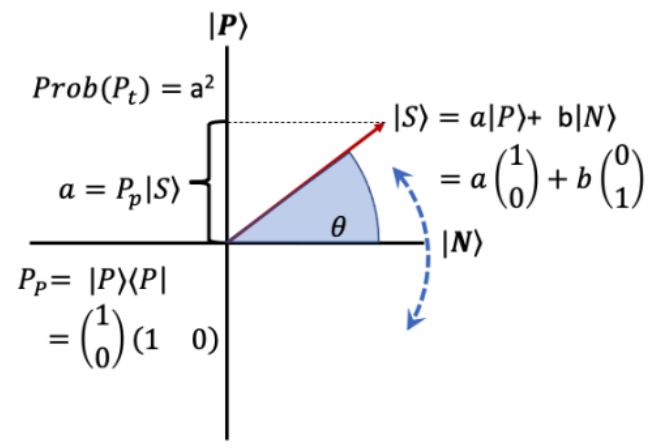

Figure 2 Hilbert space representation of decision outcomes $\mathrm{P}$ and $\mathrm{N}$. Time evolution of the amplitudes' changes is expressed with unitary transformation.

Due to the unitary evolution, the angle $\theta$ changes and results in temporal oscillation (shown in Figure 3).

The probability of a decision $\mathrm{P}$ at time $\mathrm{t}$ is calculated with the amplitude square, $a^{2}$.

Critical for QPT is, introducing the superposition principle into the decision modeling. As shown in Figure 3, the superposition principle indicates that a definite decision state does not exist in the human mind [5], [7], [18]; instead, there is a superposition of states that evolves temporally with unitary evolution (shown in Figure 3) [5], [7], [18].

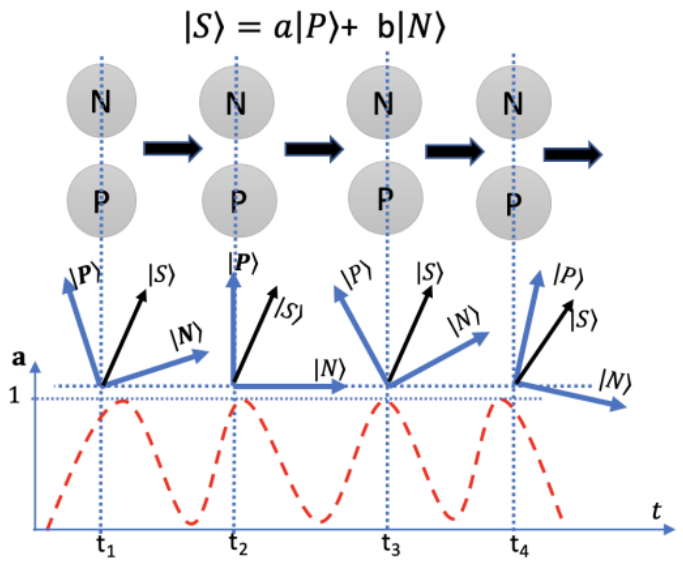

Figure 3 Representation of decision outcomes with superposition in Hilbert space and resulting temporal oscillation.

\subsection{Double-slit experiment}

To better understand the difference between the CPT and QPT, the double-slit experiment can be 
examined with both approaches. As shown in Figure 4 , an electron is fired towards a metal sheet that has two slits on it. The slits are close to each other such that electrons can pass through the slits and then be detected on the screen [19]. When both slits are open, and no detector is placed on any of the slits, an interference pattern is observed on the detector screen; if a detector is placed on any of the slits and detects an electron or one of the slits is closed, the interference pattern on the screen vanishes.

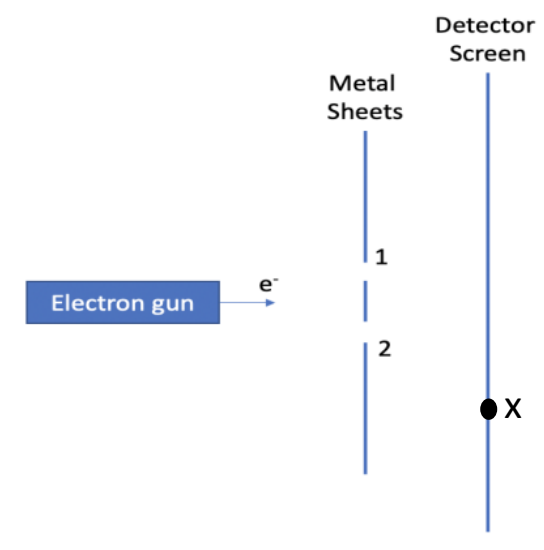

Figure 4. Double Slit Experiment with an electron gun

Now suppose slit two in Figure 4 is closed, and electrons can only pass through slit 1 . If the state of the electron is $\left|\psi_{1}\right\rangle$, then the probability distribution that the electron is detected on the detector screen at position $\mathrm{x}$ is $\mathrm{P}_{1}(\mathrm{x})=\left|\left\langle\psi_{1} \mid \mathrm{x}\right\rangle\right|^{2}$ where $|\mathrm{x}\rangle$ represents the state vector of $x$. If the state of the electron is $\left|\Psi_{2}\right\rangle$, then the probability distribution becomes $\mathrm{P}_{2}(\mathrm{x})=$ $\left|\left\langle\psi_{2} \mid x\right\rangle\right|^{2}[19]$.

In the case of having both slits are open, electrons can pass through any of them. If the electron goes through the slit 1 or 2 , then the probability distribution for the electron on the screen becomes $P_{1}(x)$ for the electrons passing through slit 1 and $\mathrm{P}_{2}(\mathrm{x})$ for the ones passing through slit 2 . Suppose half of the electrons pass through slit 1 and the other half through slit 2, the probability distribution of electrons on the screen will be [19]

$$
P(x)=\frac{1}{2} P_{1}(x)+\frac{1}{2} P_{2}(x) .
$$

After passing the metal sheet with slits, the electron is in a mixture of two states, $\left|\psi_{1}\right\rangle$ and $\left|\psi_{2}\right\rangle$, and Equation 5 represents the probability distribution for the electron. When the electron reached the detector screen, the probability distribution for being in the state $|x\rangle$ is [19]

$\mathrm{P}_{\text {mix }}(\mathrm{x})=\operatorname{Tr}[|\mathrm{x}\rangle\langle\mathrm{x}| \rho]=\frac{1}{2} \mathrm{P}_{1}(\mathrm{x})+\frac{1}{2} \mathrm{P}_{2}(\mathrm{x})=\mathrm{P}(\mathrm{x})(6)$
Where $\rho$ represents the state of the electron mix

$$
\rho=\frac{1}{2}\left(\left|\psi_{1}\right\rangle\left\langle\psi_{1}|+| \psi_{2}\right\rangle\left\langle\psi_{2}\right|\right)
$$

If Equation 7 is used to represent the state of the electron mix, the interference pattern on the detector screen cannot be explained because the actual state of the system is in a superposition [19] :

$$
\frac{1}{\sqrt{2}}\left(\left|\psi_{1}\right\rangle+\left|\psi_{2}\right\rangle\right)
$$

If Equation 8 is used, it accounts for not having any detection on any of the slits. The total probability of being in the state $|x\rangle$ becomes [19]:

$$
\mathrm{P}_{\text {tot }}(\mathrm{x})=\frac{1}{2} \mathrm{P}_{1}(\mathrm{x})+\frac{1}{2} \mathrm{P}_{2}(\mathrm{x})+\underbrace{\operatorname{Re}\left[\left\langle\mathrm{x} \mid \Psi_{1}\right\rangle\left\langle\psi_{2} \mid \mathrm{x}\right\rangle\right]}_{\text {interference term }}
$$

The interference term in Equation 9 can be either negative or positive, and explains why $\mathrm{P}_{\text {tot }}(\mathrm{x}) \neq \mathrm{P}(\mathrm{x})$. This situation changes if a detector is placed on either of the slits in Figure 4 to measure if an electron passed through the chosen slit; the interference term in Equation 8 vanishes and $\mathrm{P}_{\text {tot }}(\mathrm{x})=\mathrm{P}(\mathrm{x})$ [19].

\subsection{Double-slit experiment in a decision- making context}

Suppose Alice, a human decision-maker, and iDecide, an AI decision support tool, are on duty for an Intelligence, Surveillance, and Reconnaissance (ISR) mission. The ISR mission is to monitor a group of aliens on a far distant planet on which the U.S. Space Force's (USSF) ground units conduct an operation. According to the intelligence report, there are two categories of aliens on the planet, "Beko" and "Keko". A category "Beko" aliens are more likely to attack the USSF ground units, where "Keko" aliens are more likely to be friendly to the USFF ground units. The mission is to notify the ground units as early as possible of alien presence types so that ground forces can either withdraw "Withdraw" or attack "Attack" based on the type of approaching alien. Making the task more difficult is the aliens' cross-categorical facial features; their images can generate bistable perceptual stimuli; ergo, "Beko" could look like "Keko" vice versa. To complete this mission, Alice and Bob can interact via a platform, which includes a common operating picture (COP). iDecide provides recommendations on "Beko" and "Keko" to aid in making her decision to have the ground forces withdraw or attack. Alice interacts with iDecide using a common operating picture that provides situational awareness and information about Beko and "Keko," however interactions with ground forces are directed by Alice and do not require interaction with iDecide. 
Two scenarios are introduced. In Scenario-1, Alice decides, without interacting with iDecide or the COP, to instructs ground forces to withdraw, as shown in Figure 5a. In Scenario-2, Alice, based on the COP and $\mathrm{s}$ feedback based on iDecide inputs, identifies a Beko alien presence and instructs the ground forces to Withdraw, as shown in Figure 5b. The difference between these two scenarios is that in Scenario-1, Alice maintains her indecisiveness concerning the category of the approaching alien (as in the case of having both slits are open in the double-slit experiment); in Scenario-2, she makes her choice and resolves the indecisiveness concerning the category choice (as having a detector on one of the slits in the double-slit experiment).

These two decision processes have been tested through numerous studies demonstrating a systemic violation of the total probability for the condition in Figure 5a as it occurs in the double-slit experiment. This violation is due to the mental indecisiveness (ontic uncertainty) [20] that influences the subsequent decision in Figure 5a but does not influence the decision in Figure $5 \mathrm{~b}$ because the superposition state (as explained in Equations 8 and 9) concerning the category is resolved and hence the mental (ontic) uncertainty is resolved by making a category choice.

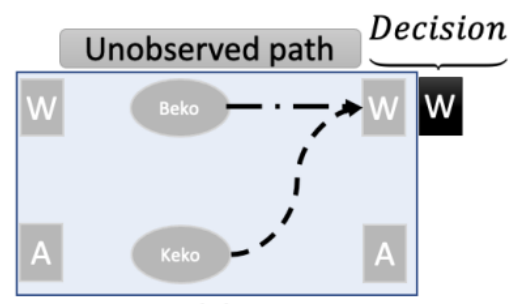

(a)

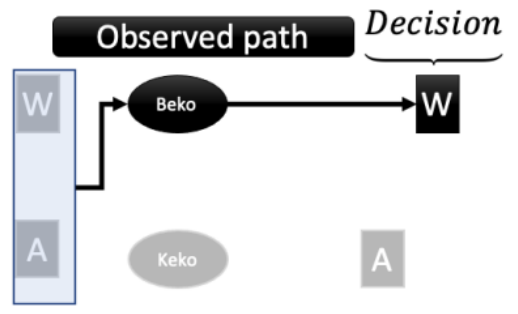

(b)

Figure 5. Path diagram representations of two decision scenarios: (a) the condition in which there is no observation, and the choice of outcome is "W"; and (b) the path that is taken is known, and the choice of outcome is "W."

There are always ontic and epistemic aspects of a system in any situation. Ontic states of a system are referents of individual descriptions of a system [21]. Epistemic states of a system describe the others' knowledge of the system's properties. These two states give rise to different types of uncertainty, ontic and epistemic. Epistemic uncertainty can be resolved by obtaining more information about the system [20][22]. However, no extra information can be obtained to reduce ontic uncertainty; it can only be resolved when the system interacts with the environment [20]. The distinction between the two can be articulated by re-visiting the ISR example. In Figure 5a, Alice is in an indefinite mental state, neither "Beko" nor "Keko," concerning the category of alien. Due to her indecisive mental state, Alice experiences a mental (ontic) uncertainty concerning the category of the alien. This mental uncertainty can only be resolved if Alice makes her choice of alien category. Therefore, in the case of Figure $5 \mathrm{a}$, since the ontic uncertainty is not resolved, it influences her subsequent decision. In Figure 5b, since the mental (ontic) uncertainty is resolved concerning the alien's category, the influence that occurs in Figure 5a vanishes. Typically, the effects of an interaction between Alice and Bob are scrutinized at the epistemic level. However, even though a decision, judgment, choice, communication, or selection of one alternative over another can allude to different meanings, each of these acts should be considered as an interaction between a human, machine, and the environment. Each interaction alters the human cognitive system as a constructive process.

\section{Quantum decision theory and relative information gain}

We used the quantum decision theory (QDT) introduced in [6] in the human-machine interaction model such that some of the aspects of ontic uncertainty can be captured. The mathematical structure of QDT captures the interference term that results from the superposition of composite situations that involves contextual effects, non-commutative subsequent decision making, and interference effects [6].

Suppose there are two decisions to choose, $\mathrm{D}_{1}$ and $\mathrm{D}_{2}$. According to QDT, the probability measure of choosing $D_{1}$ for $j^{\text {th }}$ as a function of time can be expressed as:

$$
p_{j}\left(D_{1}, t\right)=f_{j}\left(D_{1}, t\right)+q_{j}\left(D_{1}, t\right)
$$

Where $f\left(D_{1}\right)$ is the utility factor representing the classical probability contribution and $\mathrm{q}\left(\mathrm{D}_{1}\right)$ is the interference term. The interference term is constrained by the quarter-law and therefore, the non-informative priors for the average interference term are $\mathrm{q}\left(\mathrm{D}_{1,2}\right)=$ $\frac{1}{4},-\frac{1}{4}$ [6], [13], [14], [23], [24]. The sign of the interference term depends on the decision being attractive or repulsive in the situation [13]. 
In the rest of the paper, the utility factor, $\mathrm{f}_{\mathrm{j}}$, will be treated time-independent [14] and the quantum interference term depends on the information received by the agent $j, M_{j}(t)$ :

$$
q\left(D_{n}, t\right)=q_{0}\left(D_{n}\right) \exp \left(-\sum_{t=1}^{t^{\prime}} \varphi\left(t^{\prime}, t\right) \mu_{j}(t)\right)
$$

In Equation 11, $\mu_{\mathrm{j}}$ represents the information gained by a decision-making agent. In the case of two agents exchanging information, the information gain of the $\mathrm{j}^{\text {th }}$ agent can be expressed as [13], [14]:

$$
\mu_{i j}(t)=\sum_{n=1}^{N} p_{i}\left(D_{n}, t\right) \ln \frac{p_{i}\left(D_{n}, t\right)}{p_{j}\left(D_{n}, t\right)}
$$

In the case of having two alternative decisions, the information gain function shown in Equation 12 becomes

$\mu_{i j}(t)=p_{i}\left(D_{1}, t\right) \ln \frac{p_{i}\left(D_{1}, t\right)}{p_{j}\left(D_{1}, t\right)}+p_{i}\left(D_{2}, t\right) \ln \frac{p_{i}\left(D_{2}, t\right)}{p_{j}\left(D_{2}, t\right)}$

Then, by using $p_{i}\left(D_{1}, t\right)+p_{i}\left(D_{2}, t\right)=1$, one can rewrite Equation 13 as:

$$
\mu_{i j}(t)=p_{i}(t) \ln \frac{p_{i}(t)}{p_{j}(t)}+\left[1-p_{i}(t)\right] \ln \frac{1-p_{i}(t)}{1-p_{j}(t)}
$$

\subsection{Online medical information search: A case for human-machine interaction}

A story from The Cyber Effect [15] demonstrates the current state of human-machine interaction study. The story introduces Lisa, who went hiking with her friend during tick season as she and her friend were hiking and talked on various topics to include ticks and Lyme disease. Upon returning home from her hike, she became worried about ticks. After a personal examination, she finds a tick; she removes it with the information she obtained online. After removing the tick, she began searching the internet about Lyme disease and its symptoms based on her previous discussion with her friend during their hike. While trying to remain calm, she clicked from one search result to another, and after visiting various web pages, her anxiety increased. Stressed out and unable to sleep, she decided to continue her reading more about Lyme disease. Click after click as she tumbled into the medical webpages, and while she was reading about the consequences of Lyme disease, her anxiety was high. Lisa lost track of time, and her imagination ran wild, often missing relevant information during her searches that might have been comforting. Based on her frenetic searches, Lisa started to think that she had Lyme disease and ended up visiting a doctor in the morning. The doctor confirmed Lisa did not have Lyme disease but incurred costs for the unnecessary visit and contracted a virus from another patient who was visiting the same doctor's office [15].

Lisa's story's similarities with Alice and iDecide decision scenarios are as follows. To decide Lyme positive, there are symptoms (e.g., having red eyes) that need to be categorized. While searching online medical information about Lyme disease, Lisa could not make any decision for the intermediate symptoms; for example, during her internet searches, Lisa observed her eyes had become red, the red-eye symptom could be due to extended screen time she had while searching in the dark. However, in spite of this context, it was instead interpreted as a result of the tick bite. As a result, the mental indecisiveness concerning the symptom categories influences her Lyme positive belief.

However, after visiting a doctor, Lisa would learn the definite category choices for intermediate symptoms from a professional with the supported test result, and as a result, she would resolve her indecisiveness concerning the symptoms and would have a Lyme negative belief that couldn't be influenced by any mental indecisiveness concerning the intermediate symptoms.

\subsection{Relative information gain between agents}

Before the internet era, Lisa would have gone to a doctor if she did not feel well. During the visit, the doctor would ask questions concerning her health; Doctors have the training to make diagnoses and ask questions in a non-directive way [15]./ In contrast, a medical webpage is designed to provide information to the consumers but not make diagnoses.. As it occurred in Lisa's situation, due to the increased anxiety, the terms that are entered into the search engine would become more Lyme-related; thus, the engine would continue to recommend more web pages that contain Lyme disease information.

To model Lisa's situation as an interaction, first, we assume that humans interact with two types of machines. This includes the search engines she used and the web pages that are recommended and clicked by her. Due to the nature of interaction and rising anxiety, in our model, the information provided by the visited webpage at time $t$ has a higher weight in Lisa's memory than the earlier ones. Hence, the total received information by Lisa in the exponential power term in Equation 11 becomes: 


$$
\mathrm{M}_{\text {Lisa }}(\mathrm{t})=\sum_{\mathrm{t}=1}^{\mathrm{t}^{\prime}} \mu_{\text {Lisa }}(\mathrm{t}) \cdot \mathrm{t}
$$

In the case of having two human agents exchanging information, Equation 10, probability of choosing decision $\mathrm{D}_{1}$, becomes

$$
p_{j}(t+\tau)=f_{j}+q_{j}(t)
$$

Where the $\tau$ in Equation 17 is taken as 2.5 seconds in the model.

In the case of replacing one of the humans with an inanimate agent, the model's assumptions are as follows. Lisa's utility factor, $\mathrm{f}_{\mathrm{j}}$, is time-independent, and the interference factor, $q_{j}$, is time-dependent; therefore, the probability equation is still timedependent. Since search engines are optimized to find the most relevant web pages that can provide more information relevant to the terms that are searched, we suppose that there is no memory contribution for the inanimate agent and the interference term is zero, $q_{j}=$ 0 , and the utility factor is time-independent.

To express the content of a medical webpage in terms of probability measures, the model's assumptions are as follows. First, we assume that if a textual/semantic analysis of a medical webpage is conducted, a probability value can be assigned to the Lyme positive or negative; for example, probability of Lyme positive 0.7 means that the content of this webpage conveys information such that after reading the web content, the reader would think that he/she was $70 \%$ Lyme positive. Two types of probability calculation are used to model the inanimate agents' role in information exchange. The first probability value is time-invariant, which means that based on search entries, all the recommended medical pages' content has a Lyme positive probability value of 0.7. The second probability calculation, shown in Table 1, has a small random variation; as more search terms are entered, the search engine pulls information from various resources, and the probabilistic variation of the meaning of the text is \pm 0.01 .

The initial value of the interference term is $q_{j}=$ 0.20 for two reasons. First, a positive value is chosen because Lisa thought that she had Lyme disease and began searching for information about Lyme disease by using a search engine; therefore, the positive value indicates that she was attracted to any Lyme disease information. Second, due to the quarter limit [6], [13], [14], [23], [24], to avoid a very strong attraction, $q=0.20$ is chosen.
Table 1. Initial Values for the two human-machine cases.

\begin{tabular}{|c|c|c|c|c|c|c|}
\hline Term & \multicolumn{3}{|c|}{ Case 1 } & \multicolumn{3}{c|}{ Case 2 Random $\Delta=0.01$} \\
\hline $\mathrm{p}_{\text {machine }}$ & 0.70 & 0.7 & 0.7 & $0.7 \pm \Delta$ & $0.7 \pm \Delta$ & $0.7 \pm \Delta$ \\
\hline $\mathrm{p}_{\text {human }}$ & 0.85 & 0.86 & 0.89 & 0.85 & 0.86 & 0.89 \\
\hline $\mathrm{f}_{\text {machine }}$ & 0.70 & 0.70 & 0.70 & 0.70 & 0.70 & 0.70 \\
\hline $\mathrm{f}_{\text {human }}$ & 0.65 & 0.66 & 0.69 & 0.65 & 0.66 & 0.69 \\
\hline $\mathrm{q}_{\text {machine }}$ & 0.00 & 0.00 & 0.00 & 0.00 & 0.00 & 0.00 \\
\hline $\mathrm{q}_{\text {human }}$ & 0.20 & 0.20 & 0.20 & 0.20 & 0.20 & 0.20 \\
\hline
\end{tabular}

\subsection{Case 1: Time-independent machine probability}

To calculate Lisa's relative information gain, Equation 14 is used, and the webpage's relative information gain is zero. Lisa's initial probability of Lyme positive belief is 0.85 , and the probabilistic value for the content of the webpage is 0.70 . With these initial values, Lisa's belief does not demonstrate any temporal oscillation at the beginning and decreases; however, when the difference between two probability values becomes less than 0.10 , a temporal oscillation begins in Lisa's belief, as shown in Figure 6. After an extended interaction, Lisa's belief oscillation stabilizes.

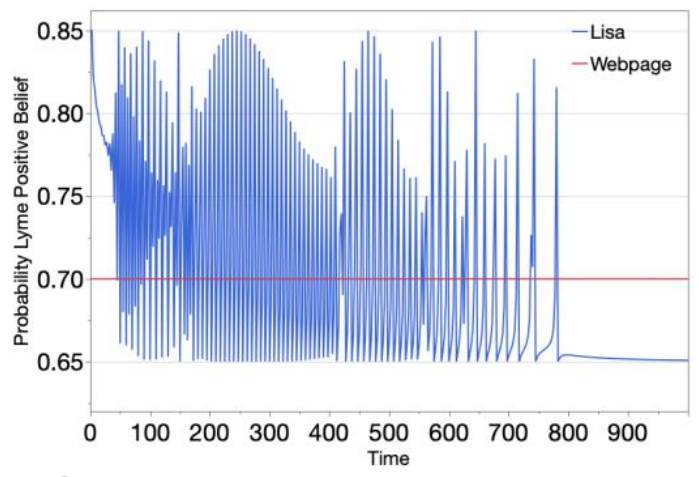

Figure 6. Temporal oscillation of probability of Lyme positive belief for Lisa; the initial probability is 0.65 .

When Lisa's initial probability Lyme positive belief becomes 0.86 , the temporal oscillation of Lisa's Lyme positive belief probability begins around the same time as in the case of 0.85 ; however, as shown in Figure 7 temporal oscillation continues, which means that metal (ontic) uncertainty continues. This means that Lisa's inability to diagnose her symptoms inflated Lisa's anxiety, indecisiveness concerning the symptoms and increased her desire to seek more information. 


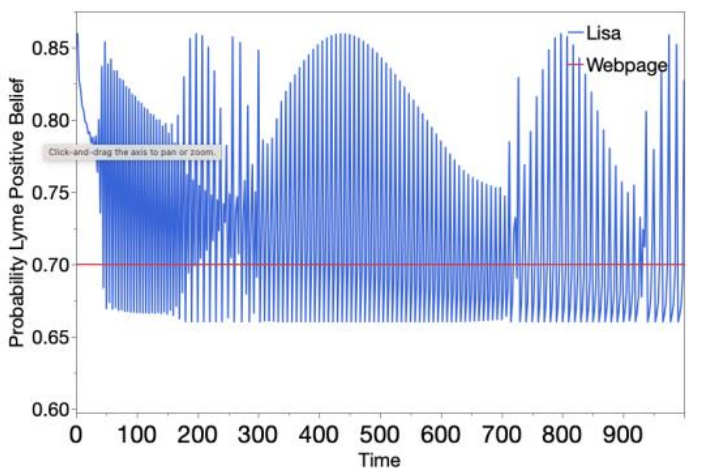

Figure 7. Temporal oscillation of probability of Lyme positive belief for Lisa; the initial probability is 0.66 .

If the initial probabilistic difference increases to 0.19 by increasing Lisa's initial utility factor value to 0.69 , as shown in Figure 8 the temporal oscillation of Lisa's Lyme positive belief probability begins earlier than the other two cases; the temporal oscillation continues such that Lisa will experience higher anxiety while interacting with the online content.

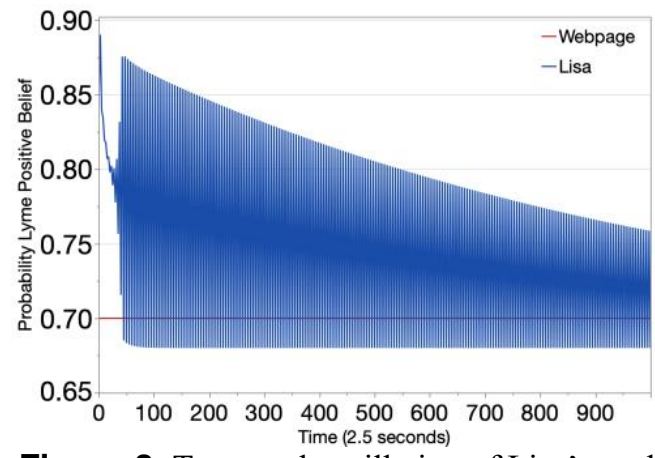

Figure 8. Temporal oscillation of Lisa's probability Lyme positive belief when the initial probability is 0.69 .

\subsection{Case 2: Webpage information content with random noise}

In case two, random noise was added to the probabilistic value of the content of the webpage that appears in the search results; in doing so, the goal is to see the effects of minute content variations in the visited webpages on Lisa's belief probability. These minute probabilistic variations are included with a random noise $\Delta=0.01$. As shown in Figure 9, temporal oscillations begin earlier than the case in which the visited web page's probabilistic representation does not vary. In the case of increasing Lisa's utility factor to 0.66 , temporal oscillation, as shown in Figure 10, begins quickly and oscillates with higher amplitude than the case shown in Figure 7; very small variations in the probabilistic value of the webpage inflates Lisa's uncertainty.

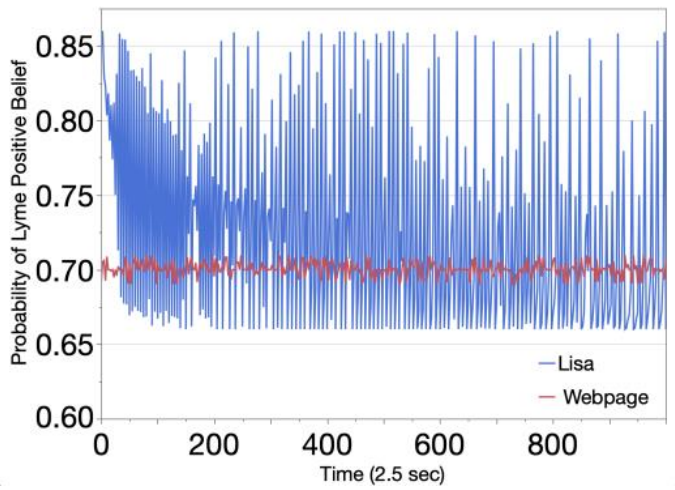

Figure 9. Temporal oscillation of probability of Lyme positive belief for Lisa; the initial probability is 0.69 .

In the case of increasing Lisa's utility factor to 0.69 , the uncertainty increases, shown in Figure 11.

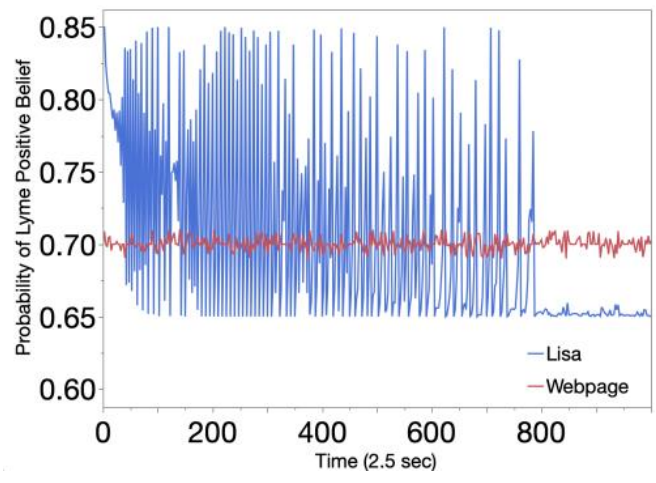

Figure 10. Temporal oscillation of probability of Lyme positive belief for Lisa.

To compare these human-machine/computer interaction scenarios with human-human interaction ones, each scenario is further studied by using Equation 14, for both agents are assumed to be humans. Suppose Lisa goes to a doctor after finding a tick on her leg without any online Lyme disease inquiry. To model these scenarios, the initial conditions are examined for two different interference factors. In the first case, both agents have positive interference factors; in the second case, Lisa has a positive interference term, and the doctor has a negative interference term.

The positive interference term represents that number of patients that are diagnosed with Lyme positive is high; therefore, the doctor thinks that this case can be a Lyme positive case. 


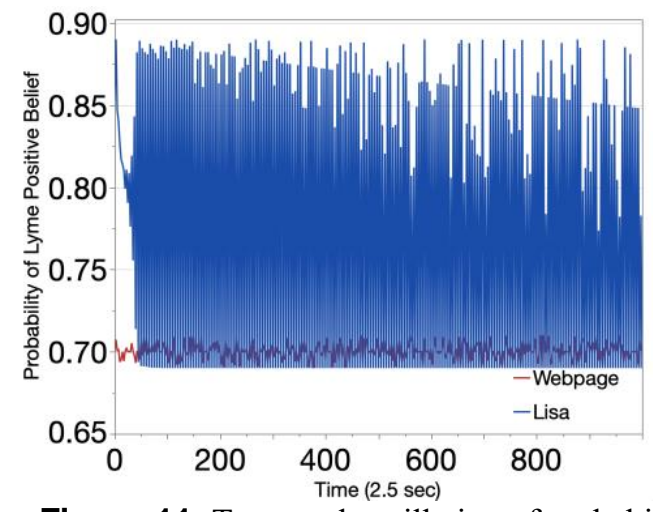

Figure 11. Temporal oscillation of probability of Lyme positive belief for Lisa.

The negative interference term represents that although it is tick season, Lyme positive cases are rare in the area. It is also assumed that since Lisa thinks she might have Lyme disease because she found a tick on her leg and her initial utility factor varies between 0.65 and 0.69 ; since the doctor is natural before meeting with the patient, the doctor's utility factor is 0.5 in all of the cases. As shown in Figure 12, Lisa does not experience any temporal oscillation while interacting with a doctor in all three initial condition values.

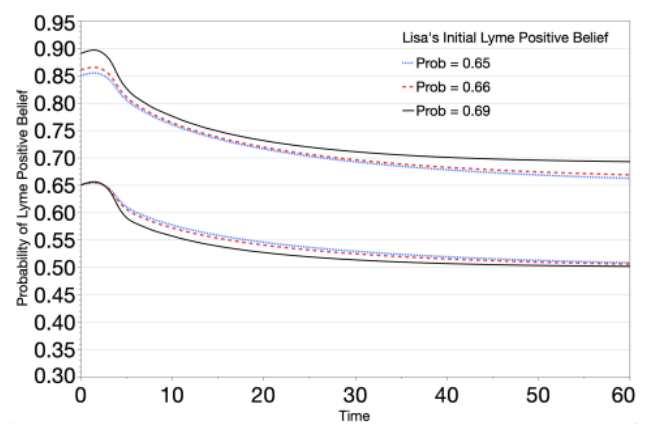

Figure 12. Temporal oscillation of probability of Lyme positive belief both doctor and patient. The asymptotic behavior represents the mental stability of the agents.

In the case of having initial disagreement due to the negative interference term of the doctor, both agents experience minute temporal oscillations (shown in Figure 13), then both agents' probabilistic understanding stabilizes to the initial utility values.

A comparison of the oscillation range for different machine probability cases are further studied for changing probability differences between agents. As shown in Figure 14, as the probabilistic difference between human and machines increases, the temporal oscillation amplitude of the human probabilities increases and scales at 0.2 .

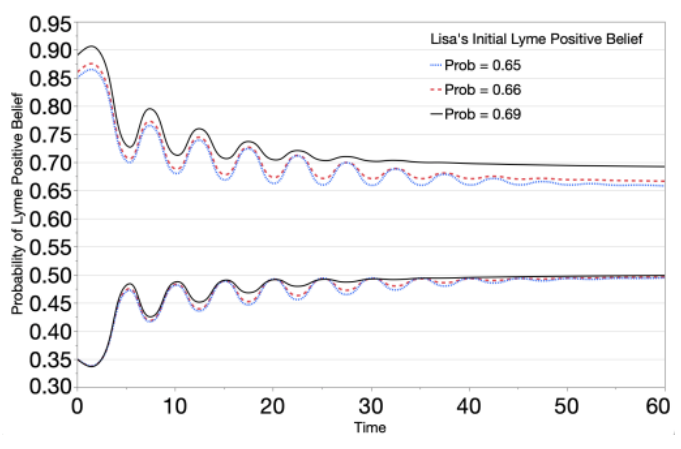

Figure 13. Temporal oscillation of probability of Lyme positive belief for both doctor and patient. After both humans experience mental uncertainty, their mental uncertainty represents asymptotic behavior.

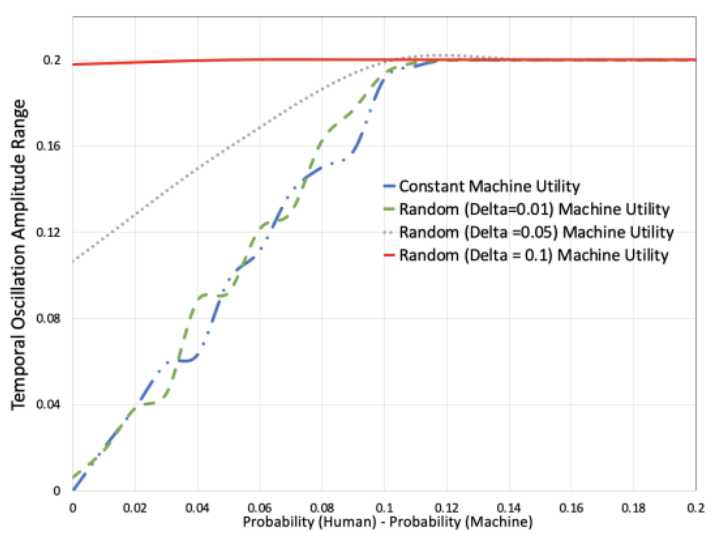

Figure 14. Temporal oscillation range (MaxMin)[Probability of Lisa's belief Lyme Positive] for different probabilistic differences between human and machine.

The reason for observing a scaling at 0.2 is due to the predetermined interference term value of 0.2 . The interesting observation is that as the noise in the machine's probabilistic representation of information increases, the scaling is observed independently from the probabilistic difference. One interpretation of this observation is that as the search engine increases the amount of available information to the human decision-maker, he/she can start experiencing higher (ontic) mental uncertainty. In return, a human can desire to seek more information and find him/herself in an information echo chamber, which can inflate anxiety.

\section{Summary and Conclusion}

This paper is intended to show how decision-related uncertainty cannot be fully explained with classical probability theory. It introduces the authors' position on the limitation of classical probability approaches 
for dealing with uncertainty. The paper illustrates this with an approach that describes a situation based on a popular case study and applying mathematical foundations from known theories to highlight the limitations of classical probability theory. This is followed by a discussion on how quantum probability theory improves the understanding of uncertainty; it is demonstrated with a scenario meant to highlight a decision process with high uncertainty to show how quantum probability theory highlights the impact of ontic uncertainty on the decision process. The paper is meant as a position using accepted theory to introduce a new perspective rather than theoretical work. It was not meant to apply a research methodology consisting of an experiment, capturing the relevant data, conducting analysis, and discussing the implications for introducing a new theory. In this paper, we modeled a situation in which an interdependency emerges between information exchanging decision-making human agents. We demonstrated that if a computer replaces one of the humans, the uncertainty that the remaining human experiences can increase.

We demonstrated that human probabilistic decision-making measures (e.g., Lyme positive belief) are affected by the minute probabilistic variation of the information that is provided by a computer (machine). The limitations of narrow AI systems that can inadvertently constraint human behavior must be comprehensively studied. As demonstrated, by modeling Lisa's situation with QPT, frenetic interactions with computers (machines) can have repercussions beyond the epistemic level.

The findings of this paper are valid only for the situations in which humans interact with computers, search engines, and online information sources. The next step in this research is to study the dynamics of information exchange between a machine with more capabilities and humans in a goal-oriented environment; this will enable to study team dynamics such as coordination, communication, and interdependency with QPT.

\section{Acknowledgement}

This research is supported by Department of the Navy, Office of Naval Research, Consortium for Robotics Unmanned Systems Education and Research at the Naval Postgraduate School.

\section{References}

[1] Department of Navy, "Department of the Navy Strategic Roadmap for Unmanned Systems (Short Version)," 2018.

[2] D. Coats and S. Gordon, "The AIM Initiative: A Strategy for Augmenting Intelligence Using Machines," 2019.

[3] S. Russell, Human Compatible Artificial Intelligence and the Problem of Control. Viking, 2019.
[4] K. Murphy, Machine Learning: A Probabilistic Perspective. Cambridge, MA: The MIT Press, 2012.

[5] J. R. Busemeyer and P. D. Bruza, Quantum Models of Cognition and Decision. Cambridge, MA, 2012.

[6] V. I. Yukalov and D. Sornette, "Decision theory with prospect interference and entanglement," Theory and Decision, vol. 70, no. 3, pp. 283-328, 2011, doi: 10.1007/s11238-010-9202-y.

[7] D. Aerts, "Quantum structure in cognition," Journal of Mathematical Psychology, vol. 53, no. 5, pp. 314-348, 2009, doi: 10.1016/j.jmp.2009.04.005.

[8] G. A. Boy, The Handbook of Human-Machine Interaction: A Human-Centered Design Approach. CRC Press, 2017.

[9] A. Leontyev, "Activity and Consciousness," Revista Dialectus, Oct. 2016, doi: 10.30611/2014n4id5173.

[10] J. Rezaei, "Best-worst multi-criteria decision-making method: Some properties and a linear model," Omega, vol. 64, pp. 126-130, Oct. 2016, doi: 10.1016/j.omega.2015.12.001.

[11] D. Baker, Guidebook to Decision-making Methods. Westinghouse Savannah River Company, 2001.

[12] Q. Zhang, V. S. S. Nadendla, S. N. Balakrishnan, and J. Busemeyer, "Strategic Mitigation of Agent Inattention in Drivers with Open-Quantum Cognition Models," pp. 1-12, 2021.

[13] V. I. Yukalov, "Evolutionary processes in quantum decision theory," Entropy, vol. 22, no. 6, p. 681, 2020, doi: 10.3390/E22060681.

[14] V. I. Yukalov, E. P. Yukalova, and D. Sornette, "Information processing by networks of quantum decisionmakers," Physica A: Statistical Mechanics and its Applications, vol. 492, pp. 747-766, 2018, doi: 10.1016/j.physa.2017.11.004.

[15] M. Aiken, The Cyber Effect: An Expert in Cyberpsychology Explains How Technology Is Shaping Our Children, Our Behavior, and Our Values--and What We Can Do About It. New York: Spiegel \& Grau, 2017.

[16] L. Savage, The Foundations of Statistics. New York: Wiley, 1954.

[17] D. Aerts, J. Broekaert, L. Gabora, and S. Sozzo, Quantum Structures in Cognitive and Social Science. 2016. doi: 10.3389/978-2-88919-876-4.

[18] Leonard Susskind and Art Friedman, Quantum Mechanics: The Theoretical Minimum. Basic Books, 2015.

[19] K. Jacobs, Quantum Measurement Theory and Its Applications. Cambridge University Press, 2014.

[20] P. Kvam, J. Busemeyer, and T. J. Pleskac, "An open system model of decision-making and temporal oscillations in preference strength," 2020.

[21] H. Atmanspacher and H. Primas, "Epistemic and ontic quantum realities," in Time, Quantum and Information., C. L. and I. O., Eds. Springer Berlin Heidelberg, 2005, pp. 301-321. doi: 10.1063/1.1874557.

[22] H. Atmanspacher, "Dynamical Entropy in Dynamical Systems," in Time, Temporality, Now, A. H. and R. E., Eds. Springer Berlin Heidelberg, 1997, pp. 327-346. doi: 10.1017/CBO9780511976155.

[23] V. I. Yukalov and D. Sornette, "Inconclusive Quantum Measurements and Decisions under Uncertainty," Frontiers in Physics, vol. 4, no. April, 2016, doi: 10.3389/fphy.2016.00012.

[24] V. I. Yukalov and D. Sornette, "Role of Information in Decision Making of Social Agents," in International Journal of Information Technology and Decision Making, 2015, vol. 14, no. 5, pp. 1129-1166. doi: $10.1142 / \mathrm{S} 0219622014500564$. 